\title{
Malignancy in giant cell tumor of bone: analysis of an open-label phase 2 study of denosumab
}

Emanuela Palmerini ${ }^{1 *}$, Leanne L. Seeger ${ }^{2}$, Marco Gambarotti ${ }^{3}$, Alberto Righi ${ }^{3}$, Peter Reichardt ${ }^{4}$, Susan Bukata ${ }^{2}$, Jean-Yves Blay ${ }^{5}$, Tian Dai ${ }^{6}$, Danielle Jandial ${ }^{6}$ and Piero Picci ${ }^{1}$

\begin{abstract}
Background: Giant cell tumor of bone (GCTB) is a rare osteoclastogenic stromal tumor. GCTB can rarely undergo malignant transformation. This post hoc analysis evaluated and classified malignancies in patients with GCTB who received denosumab.

Methods: This analysis was conducted on patients with pathologically confirmed GCTB and measurable active disease treated with denosumab $120 \mathrm{mg}$ subcutaneously once every 4 weeks, with loading doses on study days 8 and 15 , as part of a phase 2, open-label, multicenter study. We identified potential cases of malignancy related to GCTB through an independent multidisciplinary review or medical history, associated imaging or histopathologic reports, and disease course. The findings were summarized and no statistical analysis was performed.

Results: Twenty of five hundred twenty-six patients (3.8\%) who received at least one dose of denosumab were misdiagnosed with GCTB that was later discovered to be malignancies: five primary malignant GCTB, five secondary malignant GCTB, four sarcomatous transformations, and six patients with other malignancies (giant cell-rich osteosarcoma, undifferentiated pleomorphic sarcoma, spindle cell sarcoma, osteogenic sarcoma, phosphaturic mesenchymal tumor of mixed connective tissue type, and fibrosarcoma/malignant fibrous histiocytoma). Many malignancies were present before denosumab was initiated (8 definitive cases, 7 likely cases), excluding potential involvement of denosumab in these cases. Signs associated with potential misdiagnoses of GCTB included poor mineralization with denosumab treatment, rapid relapse in pain, or a failure of the typical dramatic improvement in pain normally observed with denosumab.
\end{abstract}

Conclusions: Although rare, GCTB can undergo malignant transformation, and rates in this study were consistent with previous reports. Signs of poor mineralization or lack of response to denosumab treatment may warrant close monitoring.

Trial registration: clinicaltrials.gov, (NCT00680992). Registered May 20, 2008.

Keywords: Denosumab, Giant cell tumor of bone, Bone neoplasms, RANK ligand

\footnotetext{
*Correspondence: emanuela.palmerini3@unibo.it

'Chemotherapy Unit, IRCCS Istituto Ortopedico Rizzoli, Department of

Experimental, Diagnostic and Specialty Medicine (DIMES), Bologna University,

40136 Bologna, Italy

Full list of author information is available at the end of the article
}

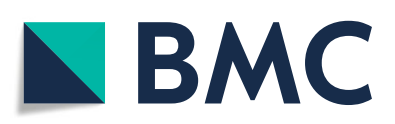

(c) The Author(s). 2021 Open Access This article is licensed under a Creative Commons Attribution 4.0 International License, which permits use, sharing, adaptation, distribution and reproduction in any medium or format, as long as you give appropriate credit to the original author(s) and the source, provide a link to the Creative Commons licence, and indicate if changes were made. The images or other third party material in this article are included in the article's Creative Commons licence, unless indicated otherwise in a credit line to the material. If material is not included in the article's Creative Commons licence and your intended use is not permitted by statutory regulation or exceeds the permitted use, you will need to obtain permission directly from the copyright holder. To view a copy of this licence, visit http://creativecommons.org/licenses/by/4.0/ The Creative Commons Public Domain Dedication waiver (http://creativecommons.org/publicdomain/zero/1.0/) applies to the data made available in this article, unless otherwise stated in a credit line to the data. 


\section{Background}

Giant cell tumor of bone (GCTB) is a rare, osteoclastogenic stromal tumor [1]. GCTB is classified as an intermediate, locally aggressive, and rarely metastasizing tumor [2-4], with occasional distant slow-growing metastases. Furthermore, GCTB can undergo malignant transformation to high-grade sarcoma, such as osteosarcoma or undifferentiated sarcoma [4]. Malignancy in GCTB is a known risk reported to occur in 1 to $4 \%$ of patients and has been categorized as primary malignant (PM) GCTB, secondary malignant (SM) GCTB, or sarcomatous transformation [5]. PMGCTB is observed at initial GCTB diagnosis as an area of highly pleomorphic mononuclear cells coexistent and adjacent to an area of otherwise conventional benign GCTB area within the same lesion; therefore, sampling error in biopsies can be associated with missed diagnosis of PMGCTB [6]. Differential diagnoses of PMGCTB include giant cell-rich osteosarcoma, benign GCTB, aneurysmal bone cysts, chondroclastomas, and brown tumor of bone [7]. SMGCTB occurs at the site of a previously treated benign GCTB lesion and the pre-existing GCTB may be evident. SMGCTB is most commonly associated with prior radiation therapy [8], and is best considered as post-radiation sarcoma. However, SMGCTB may also occur after exclusive surgical treatment in the absence of prior radiotherapy [9]. Such cases are described as sarcomatous transformation of a previously documented benign GCTB. Misdiagnosis of GCTB, instead of PMGCTB or SMGCTB, can lead to improper treatment of an aggressive disease with typically poor prognosis [7].

Denosumab is a fully human RANKL inhibitor approved for use in adults and skeletally mature adolescents with GCTB that is unresectable or when surgical resection is likely to result in severe morbidity [10]. The purpose of this analysis was to evaluate and classify malignancies in patients with GCTB who were treated with denosumab as part of an open-label, phase 2 study [11].

\section{Methods}

\section{Study design and patient population}

The design, methods and results of the primary analysis of this phase 2, international, multicenter, open-label study (NCT00680992) have been published [11]. Briefly, eligible patients were adults and skeletally mature adolescents at least 12 years of age with pathologically confirmed GCTB and measurable active disease within 1 year of enrollment. Patients had Eastern Cooperative Oncology Group Performance Status score $\leq 2$, no current use of alternative GCTB therapies, or known or suspected diagnosis of sarcoma, non-GCTB giant cell-rich tumors, brown cell tumor of bone, or Paget disease. Patients received $120 \mathrm{mg}$ denosumab subcutaneously once every 4 weeks, with loading doses on study days 8 and 15. The study was approved by each site's independent ethics committee (Additional file 1), and all adult patients provided written informed consent. For adolescents, written informed consent was required to be provided by the patient's parent or legal representative, and assent of the adolescent obtained if requested by the ethics committee.

\section{Assessments}

The purpose of this analysis was to examine potential cases of malignancy in GCTB. Potential malignancy cases were identified with an independent multidisciplinary review of all adverse events using the search term "neoplasms benign, malignant and unspecified (including cysts and polyps)", which were then manually reviewed for clinical confirmation. We reviewed medical history, associated imaging or histopathologic reports, and disease course for all patients who met the search criteria, as well as those who discontinued study due to disease progression. We conducted an independent multidisciplinary review of trial reports of malignancy in GCTB using a panel of seven experts with extensive experience in bone sarcomas and GCTB (pathologist, radiologist, and medical and surgical oncologists were included). De-identified pathology, imaging, and medical history were systematically collected for review. Sites were requested to provide pathology and imaging samples, if available, from three time points: initial GCTB diagnosis, pre-enrollment biopsy, and malignant diagnosis. When pathology samples or imaging was not available, records were supplemented with the local transcribed imaging and pathologic reports.

The pathologist reviewed all submitted specimens (imaging was also provided); whenever possible, additional immunohistochemical stains and molecular testing were performed (see Additional file 2, Additional file 3). Each case was then reviewed in detail, including GCTB disease history, disease chronology including prior recurrences and therapies, timing of denosumab treatment, and occurrence of malignancy. The pathologist and radiologist presented their key findings, selected representative pathologic and radiographic key images and opinions to the panel, and consensus opinions were made for each patient.

Cases were classified as PMGCTB if review showed that malignancy was present at the time of diagnosis; SMGCTB was generally classified as malignancy occurring at the site of a previously treated benign GCTB lesion, typically, but not exclusively occurring after radiotherapy. Sarcomatous transformation was reserved for cases that could have truly resulted from potential exposure to denosumab. Patient numbers and age ranges, instead of age at treatment, were assigned for the purposes of this publication only and do not link to 
patients. The findings from the expert panel were summarized; no statistical analysis was performed.

\section{Results \\ Patients}

A total of 532 patients (including 28 adolescents) were enrolled in the treatment phase (Fig. 1) and 526 patients received at least one dose of denosumab.

Patients identified with malignancies had baseline characteristics similar to the full study population, but were older (Table 1); the median (range) age was 52 (21-82) years in this analysis and 33 (13-83) years in the full study.

There were 20 cases of malignancy during the study: five PMGCTB cases, five SMGCTB cases, four sarcomatous transformations, and six other misdiagnoses. Malignancy cases included femur $(n=8)$, sacrum $(n=3)$, tibia $(n=2)$, pelvis, metatarsal, tibia, pubic ramus, lung, lung and navicular or cuneiform bones of foot, and humerus ( $n=1$ for each). A summary of the clinical courses of patients who developed malignancies during the primary study is presented in Fig. 2.

\section{Immunohistochemistry}

Most patients (15/20, 75\%) had some immunohistochemistry results (Table 2). Of the seven patients with both pre- and post-denosumab immunohistochemistry results, changes were observed in MDM2 expression (increased in six patients, decreased in one patient, unchanged in one patient), P53 (increased nuclear positivity in mononuclear cells for three patients, unchanged for three patients, decreased for one); P63 (decreased nuclear positivity in mononuclear cells in two, increased in one, unchanged in four patients). Six patients had H3F3A results: four were positive pre- denosumab and post-denosumab, and two were positive pre-denosumab but had no post-denosumab measurement, and two were negative pre-denosumab but with no post-denosumab measurement (one PMGCTB and one misdiagnosis). Regarding pre-treatment samples, 12 cases were available for pathological revision, consisting of four biopsies and eight whole sections relative to $\mathrm{cu}$ rettages or emiresections.osteogenic sarcoma and fibroblastic

FISH analysis for $M D M 2$ gene amplification was feasible in five samples; one case showed MDM2 amplification. Most of the non-informative samples were characterized by poor fixation or excessive decalcification. A weak fluorescence signal in two other samples was found in tissue of poor quality (very old tissue blocks).

Typical responses of GCTB to denosumab treatment lead to bone formation or mineralization (Fig. 3). Six of nine $(67 \%)$ patients with imaging for expert review showed decreased mineralization, which would otherwise be expected in response to denosumab: three PMGCTB, two sarcomatous transformations, and one misdiagnosis.

\section{PMGCTB}

PMGCTB was determined for five patients, three of which showed a pattern of imaging demonstrating poor mineralization. The malignant components in primary GCTB were undifferentiated pleomorphic sarcoma $(n=3)$, osteogenic sarcoma and fibroblastic osteosarcoma ( $n=1$ each) (Table 3 ). Durations of denosumab treatment ranged from 6 months to 29 months. The latency from initial diagnosis to PMGCTB diagnosis ranged from 10 months to 51 months. One patient (Patient 2) had a pathologic fracture at presentation. PMGCTB cases were characterized by a short latency period together with

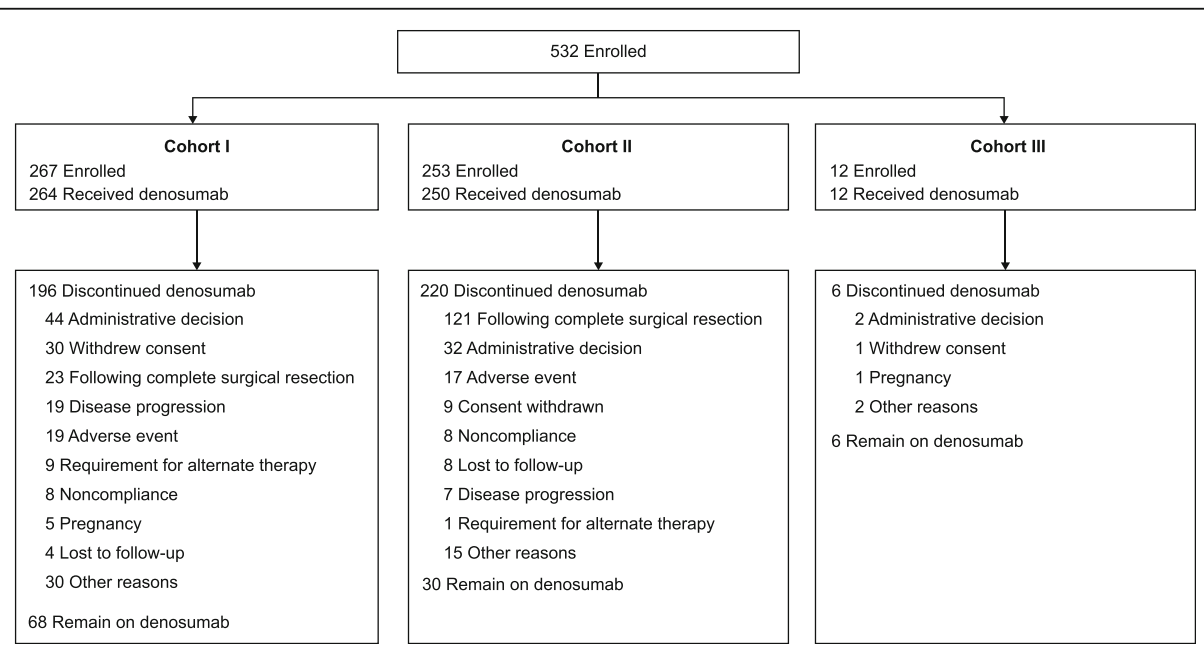

Fig. 1 Profile of the randomized controlled trial. Patients were divided into three cohorts: patients with surgically unsalvageable tumors (Cohort I), patients with surgically salvageable tumors (Cohort II), and patients rolled over from a previous study (Cohort III) 
Table 1 Baseline demographics and characteristics ${ }^{a}$

\begin{tabular}{|c|c|c|}
\hline & $\begin{array}{l}\text { All patients } \\
(N=532)\end{array}$ & $\begin{array}{l}\text { Malignancy patients } \\
(n=20)\end{array}$ \\
\hline Women & $301(57)$ & $12(60)$ \\
\hline Adolescents & $28(5)$ & 0 \\
\hline Age, median (Q1, Q3), y & $33(25,45)$ & $52(32,65)$ \\
\hline Follow-up, median (Q1, Q3), mo & $58(34,74)$ & $48(17,81)$ \\
\hline No. of denosumab doses received, median (Q1, Q3) & $34(18,61)$ & $22(11,40)$ \\
\hline \multicolumn{3}{|l|}{ GCTB disease type } \\
\hline Primary resectable & $168(32)$ & $6(30)$ \\
\hline Primary unresectable & $94(18)$ & $2(10)$ \\
\hline Recurrent resectable & $85(16)$ & $2(10)$ \\
\hline Recurrent unresectable & $185(35)$ & $10(50)$ \\
\hline \multicolumn{3}{|l|}{ Prior GCTB surgery } \\
\hline Yes & $276(52)$ & $12(60)$ \\
\hline No & $256(48)$ & $8(40)$ \\
\hline \multicolumn{3}{|l|}{ Prior GCTB radiotherapy } \\
\hline Yes & $52(10)$ & $4(20)$ \\
\hline No & $480(90)$ & $16(80)$ \\
\hline
\end{tabular}

GCTB giant cell tumor of bone, mo months, $y$ years

${ }^{a}$ Data are $\mathrm{n}(\%)$ unless indicated otherwise

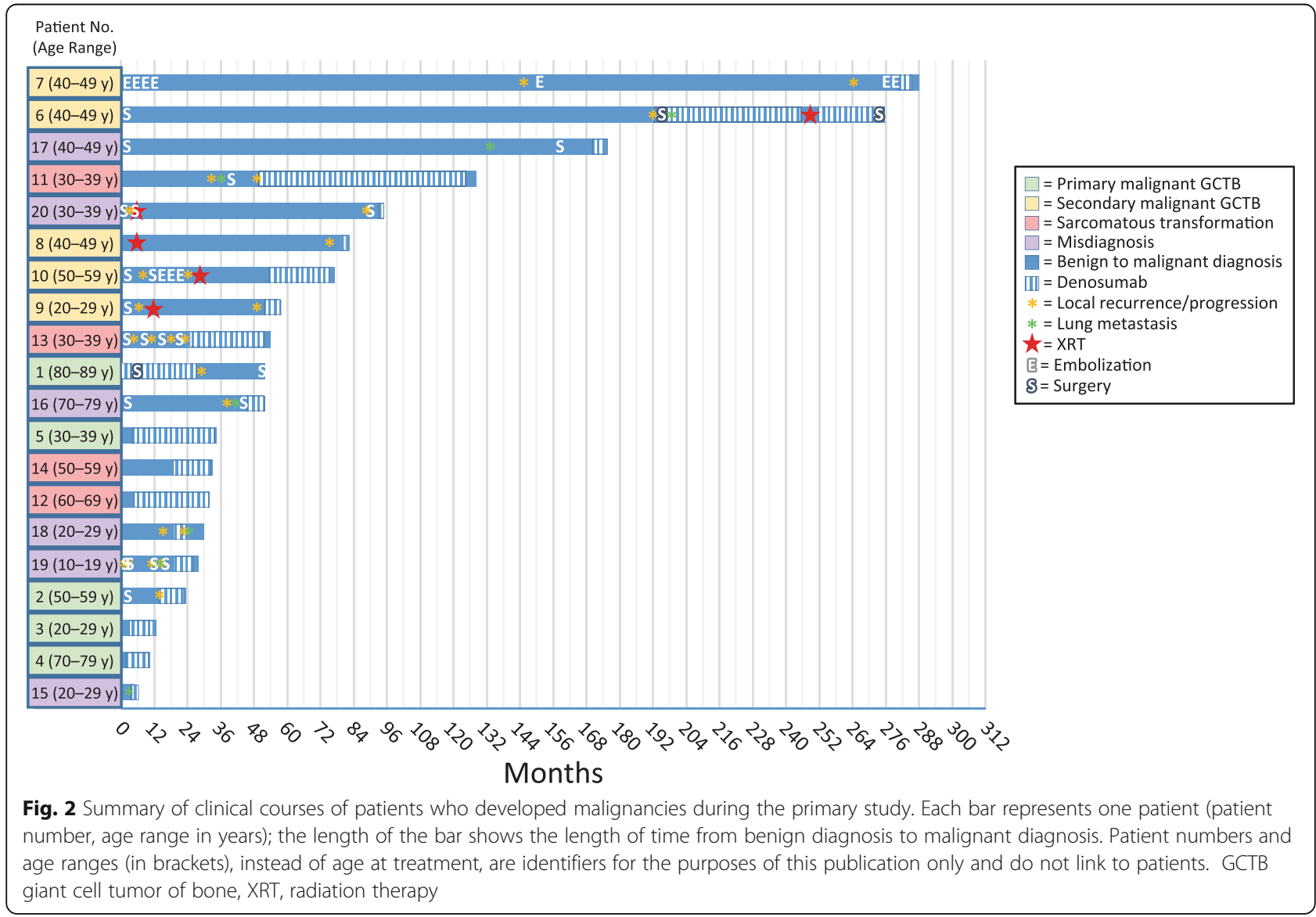


Table 2 Immunohistochemistry

\begin{tabular}{llll}
\hline $\begin{array}{l}\text { Patient } \\
\text { No. }\end{array}$ & Histology & Immunohistochemistry & H3F3A results \\
\cline { 2 - 3 } (Age & Pre-denosumab & Post-denosumab \\
Range) & & \\
\hline
\end{tabular}

\section{Primary GCTB}

1 Undifferentiated

(80- pleomorphic sarcoma

89 y)

2 Undifferentiated spindle

(50- cell sarcoma

59 y)

3 (20-

29 y)

$4 \quad$ Undifferentiated

(70- pleomorphic sarcoma

$79 \mathrm{y})$

5 Undifferentiated

(30- pleomorphic sarcoma

39 y)

\section{Secondary GCTB}

6

(40-

$49 \mathrm{y})$

(40- $\quad$ pleomorphic sarcoma

49 y)

8 Undifferentiated

(40- pleomorphic sarcoma

$49 \mathrm{y})$

$9 \quad$ Giant cell tumor with

(20- suspect progression to

29 y) sarcoma

10 High-grade

(50- undifferentiated spindle

59 y) cell sarcoma

\section{Sarcomatous transformation}

11 Undifferentiated spindle

(30- cell sarcoma

39 y)

12 High-grade

(60- osteosarcoma

69 y)

13 Undifferentiated spindle

(30- cell sarcoma

$39 \mathrm{y})$

14 High-grade

(50- osteosarcoma

$59 \mathrm{y})$

\section{Misdiagnoses}

15 Giant cell-rich

(20- osteosarcoma

$29 \mathrm{y})$

16 Pleomorphic

(70- rhabdomyosarcoma

79 y)
NA

NA

$\mathrm{P} 63^{+}, \mathrm{P}^{-} 3^{-}, \mathrm{MDM2}^{+/-}$(FISH ${ }^{\text {not }} \mathrm{P}^{-} 3^{-}, \mathrm{P}^{-} 3^{-}, \mathrm{MDM}^{+}$(FISH ${ }^{\text {not amplified }}$ )

amplified)

KI67/MIB-1 40\%, focally high, NA PDGFR- $\beta^{+}$, PDGFR- ${ }^{-}$

NA

$\mathrm{MDM2}^{-}$(FISH $\left.{ }^{\text {not available }}\right), \mathrm{P53}^{-}, \mathrm{P}^{-} 3^{-}, \mathrm{SATB}^{-}$

NE

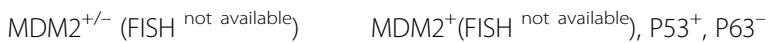

NE $\mathrm{P53}^{-}, \mathrm{P} 63^{-}$

NA

NA

NA

Vimentin ${ }^{+}, \mathrm{P} 63^{+}, \mathrm{CD}^{\circ} 1^{-}$,

NA

NA

CD34- CKCAM5.2- ${ }^{-}$, AE1 ${ }^{-} / \mathrm{AE} 3^{-}$

$\mathrm{SMA}^{-},{\mathrm{S} 100^{-}}^{-}$and desmin

NA

$\mathrm{MDM}^{+/-}\left(\mathrm{FISH}^{\text {not available }}\right), \mathrm{P}^{+/-}, \mathrm{P}^{-} 3^{-}$

NA

$\mathrm{MDM2}^{+/-}\left(\mathrm{FISH}^{\text {not available }}\right)$ NA

$\mathrm{P}_{53}^{+/-}, \mathrm{P} 63^{+/-}, \mathrm{SATB}^{+/-}$

$\mathrm{MDM2}^{+}\left(\mathrm{FISH}{ }^{\text {not available }}\right)$,

$\mathrm{P}_{53}, \mathrm{P}^{-} 3^{+/-}, \mathrm{SATB}^{+/-}$

In recurrences: $\mathrm{MDM}^{-}$(FISH

not available), $\mathrm{P} 53^{-}, \mathrm{P} 63^{-}$

$\mathrm{SATB}^{+/-}$

$\mathrm{MDM}^{+/-}\left(\mathrm{FISH}^{\text {not available }}\right), \mathrm{P}^{+} 3^{+}, \mathrm{P} 63^{+/-}, \mathrm{SATB}^{-}$

$\mathrm{H}_{3} \mathrm{~F} 3 \mathrm{~A}^{+}$

Pre-denosumab: $\mathrm{H}_{3} \mathrm{~F}^{+} \mathrm{A}^{+}$(at initial diagnosis and recurrences)

NA

$\mathrm{P}^{-} 3^{-} \mathrm{P}^{+/-}, \mathrm{MDM}^{-}$(FISH $^{\text {not }} \mathrm{P}^{-} 3^{-}, \mathrm{P}^{+} 3^{+}, \mathrm{MDM}^{+}$(FISH $\left.{ }^{\text {not available }}\right)$

available);

$\mathrm{P63}^{+}, \mathrm{P} 53^{+}, \mathrm{MDM}^{+}\left(\mathrm{FISH}^{\text {not }} \mathrm{P}^{-}, \mathrm{P}^{-} 3^{+}, \mathrm{MDM}^{+}\right.$(FISH ${ }^{\text {not available }}$ )

Pre-denosumab: ${\mathrm{H} 3 \mathrm{~F} 3 \mathrm{~A}^{+}}^{+}$

Post-denosumab: $\mathrm{H}_{3} \mathrm{~F} 3 \mathrm{~A}^{+}$

$\mathrm{P53}^{+}, \mathrm{MDM2}^{+}$(by FISH ${ }^{\text {amplified}}$ ) at malignant diagnosis but MDM2 ${ }^{-}$(FISH ${ }^{\text {not amplified) at }}$ NA initial GCTB diagnosis

$\mathrm{MDM}^{+/-}\left(\mathrm{FISH}{ }^{\text {not available }}\right.$, $\mathrm{P}^{2} 3^{+/-}, \mathrm{P} 63^{-}$

$\mathrm{MDM}^{+}\left(\mathrm{FISH}^{\text {not available }}\right), \mathrm{P}^{-} 3^{-}, \mathrm{P}^{+/-} 3^{+-} \mathrm{SATB}^{+}$

Pre-denosumab: $\mathrm{H} 3 \mathrm{~F} 3 \mathrm{~A}^{+}$
NA

$\mathrm{P} 63^{-}, \mathrm{P}^{2} 3^{+}, \mathrm{MDM}^{+/-}$
available)
$\mathrm{SMA}^{+/-}$, S100-, Ki67/MIB1 20\%CD68 ${ }^{+}$, vimentin ${ }^{+}$, focally positive for CD45 and SMA, S100-, CD30- CD15', Ki67 showed moderately high proliferative index

$\mathrm{P63}^{-}, \mathrm{P53}^{+}, \mathrm{MDM}^{+}$(by FISH ${ }^{\text {not amplified }}$ ), desmin ${ }^{+}$, myogenin $^{+}$
NA

Pre-denosumab: H3F $3 A^{-}$ 
Table 2 Immunohistochemistry (Continued)

\begin{tabular}{|c|c|c|c|c|}
\hline \multirow{2}{*}{$\begin{array}{l}\text { Patient } \\
\text { No. } \\
\text { (Age } \\
\text { Range) }\end{array}$} & \multirow[t]{2}{*}{ Histology } & \multicolumn{2}{|c|}{ Immunohistochemistry } & \multirow[t]{2}{*}{$\mathrm{H} 3 \mathrm{~F} 3 \mathrm{~A}$ results } \\
\hline & & Pre-denosumab & Post-denosumab & \\
\hline $\begin{array}{l}17 \\
(40- \\
49 y)\end{array}$ & $\begin{array}{l}\text { Undifferentiated spindle } \\
\text { cell sarcoma }\end{array}$ & \multicolumn{2}{|c|}{ CK AE1/AE3CD68 ${ }^{+}$, vimentin ${ }^{++}$, cytokeratin AE $1 / 3^{+},{\mathrm{S} 100^{-}}^{-}$} & NA \\
\hline $\begin{array}{l}18 \\
(20- \\
29 y)\end{array}$ & $\begin{array}{l}\text { Osteogenic sarcoma } \\
\text { (present pre-enrollment) }\end{array}$ & NA & NA & NA \\
\hline $\begin{array}{l}19 \\
(10- \\
19 y)\end{array}$ & $\begin{array}{l}\text { Phosphaturic } \\
\text { mesenchymal tumor of } \\
\text { mixed connective tissue } \\
\text { type }\end{array}$ & NA & NA & NA \\
\hline $\begin{array}{l}20 \\
(30- \\
39 y)\end{array}$ & $\begin{array}{l}\text { Undifferentiated spindle } \\
\text { cell sarcoma }\end{array}$ & NA & NA & NA \\
\hline
\end{tabular}

CK cytokeratin, FISH fluorescence in situ hybridization, GCTB giant cell tumor of bone, MDM2 mouse double minute 2, NA not available, NE not evaluable, PDGFR platelet-derived growth factor receptors, SATB2 special AT-rich sequence-binding protein 2, SMA smooth muscle antibody

${ }^{a}$ Patient numbers and age ranges (in brackets), instead of age at treatment, are identifiers for the purposes of this publication only and do not link to patients

observations of poor responses to denosumab either on imaging or pain control, with progressive and aggressive clinical courses. For most of these patients, review of available baseline histology confirmed the presence of PMGCTB before enrollment and start of denosumab treatment (Fig. 4a).

\section{SMGCTB}

Five of 20 patients with malignancy developed SMGC $\mathrm{TB}$, four after previous radiotherapy (Table 3). Compared with PMGCTB, patients with SMGCTB had longer latency from initial GCTB diagnosis, ranging from 56 months up to 25 years. Duration of denosumab treatment ranged from 1 month to 75 months. For four patients, malignancy was definitively present (Patients 8 and 10) or likely present before denosumab treatment, as verified by re-examination of pathology samples or report (Fig. 4b). A malignancy was classified as likely, but not definitively present, before denosumab, when there was a lack of sufficient biopsies for expert review (Patients 7 and 9). Patient 6 developed a malignancy after radiotherapy and denosumab treatment, but did not have preenrollment pathology available for review.

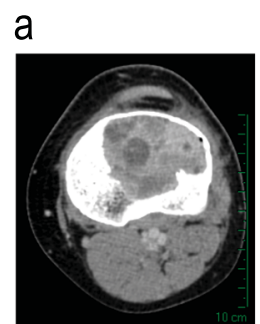

Pre-denosumab

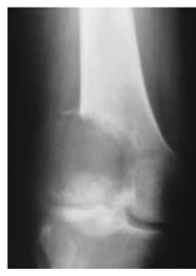

Pre-denosumab

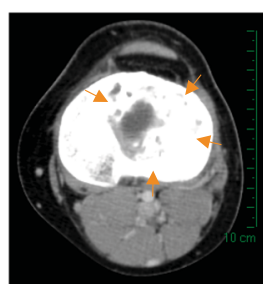

12 months post-denosumab

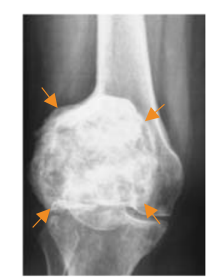

8 months post-denosumab

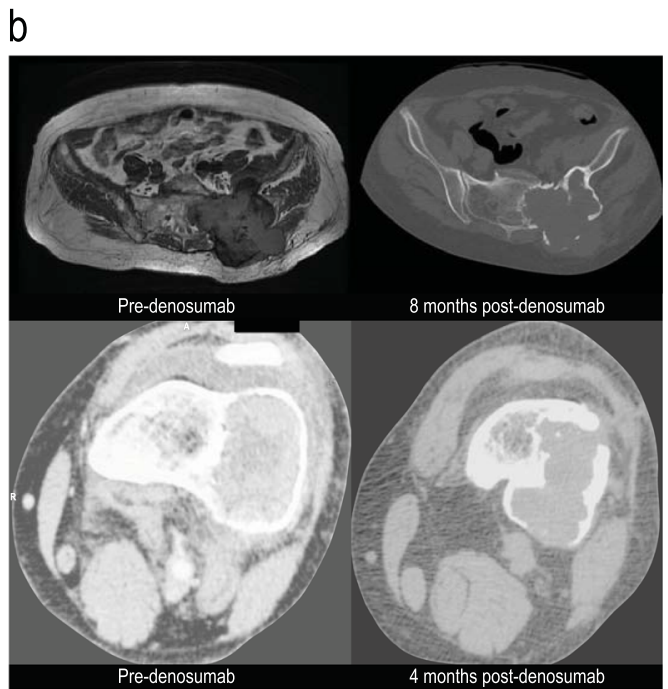

Fig. 3 Misdiagnosis of GCTB. a Typical response of GCTB to denosumab leads to bone formation and calcification (top two images are axial CT soft tissue window and bottom two images are anteroposterior radiographs). $\mathbf{b}$ In misdiagnosed PMGCTB, poor calcification in response to denosumab is shown (top two images are axial T1-weighted MRI, axial CT bone windows and bottom two are axial CT soft tissue windows) 
Table 3 Malignancies in giant tumor of bone

\begin{tabular}{|c|c|c|c|c|c|c|c|c|}
\hline $\begin{array}{l}\text { Patient no. } \\
\text { (age range) }\end{array}$ & Cohort & Site & Malignant histology & $\begin{array}{l}\text { Radiation } \\
\text { received, } \\
\text { dose }\end{array}$ & $\begin{array}{l}\text { Latency } \\
\text { period }\end{array}$ & $\begin{array}{l}\text { Duration of } \\
\text { denosumab }\end{array}$ & $\begin{array}{l}\text { Malignancy } \\
\text { present } \\
\text { prior to }\end{array}$ & $\begin{array}{l}\text { Malignancy } \\
\text { outcome }\end{array}$ \\
\hline
\end{tabular}

\begin{tabular}{|c|c|c|c|c|c|c|c|c|}
\hline \multicolumn{9}{|c|}{ Primary malignancies } \\
\hline $1(80-89$ y) & $\begin{array}{l}\text { Primary } \\
\text { resectable }\end{array}$ & Femur & $\begin{array}{l}\text { Initial diagnosis: appearance } \\
\text { suspicious of sarcoma based } \\
\text { on imaging atypical for GCTB } \\
\text { and no lytic bone lesions } \\
\text { Diagnosis: Undifferentiated } \\
\text { pleomorphic sarcoma }\end{array}$ & No & $\begin{array}{l}4 y, 3 \\
\text { mo }\end{array}$ & $2 \mathrm{y}, 5 \mathrm{mo}$ & Likely $^{\mathrm{b}}$ & $\begin{array}{l}\text { Death after } \\
\text { postsurgical } \\
\text { complications from } \\
\text { femur replacement }\end{array}$ \\
\hline $2(50-59$ y) & $\begin{array}{l}\text { Recurrent } \\
\text { unresectable }\end{array}$ & Femur & $\begin{array}{l}\text { Giant cell tumor with } \\
\text { pleiomorphic spindle cells, } \\
\text { suspect malignant areas, } \\
\text { extensive reactive changes } \\
\text { due to fracture } \\
\text { Diagnosis: Undifferentiated } \\
\text { spindle cell sarcoma }\end{array}$ & No & $2 y$ & $10 \mathrm{mo}$ & Likely $^{\mathrm{b}}$ & $\begin{array}{l}\text { Chemotherapy with } \\
\text { complete response }\end{array}$ \\
\hline $3(20-29$ y) & $\begin{array}{l}\text { Primary } \\
\text { resectable }\end{array}$ & Pelvis & $\begin{array}{l}\text { Numerous cores, largest was } 1.5 \\
\mathrm{~cm} \text {, consisting of brown, friable } \\
\text { tissue; GCTB with atypical features } \\
\text { and high expression of } \\
\text { proliferation markers } \\
\text { Diagnosis: Osteogenic sarcoma }\end{array}$ & No & $13 \mathrm{mo}$ & $6 \mathrm{mo}$ & Likely $^{\mathrm{b}}$ & Lost to follow-up \\
\hline 4 (70-79 y) & $\begin{array}{l}\text { Primary } \\
\text { resectable }\end{array}$ & Sacrum & $\begin{array}{l}\text { Spindle cell proliferation and } \\
\text { cellular atypia, no giant cells. } \\
\text { Suggests malignant } \\
\text { transformation characterized } \\
\text { by aspects of pleomorphism } \\
\text { and cellular atypia with mitotic } \\
\text { index elevated } \\
\text { Diagnosis: Undifferentiated } \\
\text { pleomorphic sarcoma }\end{array}$ & No & $10 \mathrm{mo}$ & $8 \mathrm{mo}$ & Yes & $\begin{array}{l}\text { Death of primary } \\
\text { disease } 3 \text { mo after } \\
\text { malignancy } \\
\text { diagnosis }\end{array}$ \\
\hline $5(30-39 y)$ & $\begin{array}{l}\text { Primary } \\
\text { resectable }\end{array}$ & Tibia & $\begin{array}{l}\text { Malignant spindle cell sarcoma } \\
\text { (no maturation due to } \\
\text { denosumab) } \\
\text { Diagnosis: Undifferentiated } \\
\text { pleomorphic sarcoma }\end{array}$ & No & $\begin{array}{l}2 y, 8 \\
\text { mo }\end{array}$ & $2 \mathrm{y}, 4 \mathrm{mo}$ & Yes & $\begin{array}{l}\text { Patient underwent } \\
\text { planned amputation } \\
\text { plus chemotherapy }\end{array}$ \\
\hline \multicolumn{9}{|l|}{ SMGCTB } \\
\hline $6(40-49 y)$ & $\begin{array}{l}\text { Recurrent } \\
\text { unresectable }\end{array}$ & Metatarsus & Diagnosis: High-grade sarcoma & Yes, 50 Gy & $13 y$ & $6 \mathrm{y}, 3 \mathrm{mo}$ & No & $\begin{array}{l}\text { Amputation plus } \\
\text { chemotherapy; } \\
\text { deceased } 12 \text { mo } \\
\text { after malignancy } \\
\text { diagnosis }\end{array}$ \\
\hline $7(40-49 y)$ & $\begin{array}{l}\text { Recurrent } \\
\text { unresectable }\end{array}$ & Sacrum & $\begin{array}{l}\text { At enrollment, lesion } \\
\text { contained few giant cells. In } \\
\text { foci, sheets of cells exhibiting } \\
\text { epithelioid morphology with } \\
\text { cytologic atypical and brisk } \\
\text { mitotic activity, including } \\
\text { atypical mitoses } \\
\text { Diagnosis: Undifferentiated } \\
\text { pleomorphic sarcoma }\end{array}$ & No & $25 y$ & $4 \mathrm{mo}$ & Likely $^{\mathrm{b}}$ & $\begin{array}{l}\text { Deceased } 6 \text { mo after } \\
\text { malignancy } \\
\text { diagnosis }\end{array}$ \\
\hline $8(40-49 y)$ & $\begin{array}{l}\text { Recurrent } \\
\text { resectable }\end{array}$ & Tibia & $\begin{array}{l}\text { Malignant spindle cell sarcoma } \\
\text { (no maturation due to } \\
\text { denosumab) } \\
\text { Diagnosis: Undifferentiated } \\
\text { pleomorphic sarcoma }\end{array}$ & Yes, 56 Gy & $\begin{array}{l}7 y, 8 \\
\text { mo }\end{array}$ & $1 \mathrm{mo}$ & Yes & $\begin{array}{l}\text { Amputation plus } \\
\text { chemotherapy; alive } \\
\text { at last follow-up }\end{array}$ \\
\hline $9(20-29 y)$ & $\begin{array}{l}\text { Recurrent } \\
\text { resectable }\end{array}$ & Femur & $\begin{array}{l}\text { Areas of solid ABC and GCTB } \\
\text { associated with epithelial and } \\
\text { spindle cell proliferation. } \\
\text { Diagnosis: GCTB with suspect } \\
\text { progression to sarcoma }\end{array}$ & Yes, 56 Gy & $\begin{array}{l}4 y, 8 \\
\text { mo }\end{array}$ & $6 \mathrm{mo}$ & Likely $^{\mathrm{b}}$ & $\begin{array}{l}\text { Lung metastases } \\
\text { diagnosed soon after } \\
\text { femur malignancy; } \\
\text { deceased } 5 \text { mo after } \\
\text { malignancy } \\
\text { diagnosis }\end{array}$ \\
\hline
\end{tabular}


Table 3 Malignancies in giant tumor of bone (Continued)

\begin{tabular}{|c|c|c|c|c|c|c|c|c|}
\hline $\begin{array}{l}\text { Patient no. } \\
\text { (age range) }^{a}\end{array}$ & Cohort & Site & Malignant histology & $\begin{array}{l}\text { Radiation } \\
\text { received, } \\
\text { dose }\end{array}$ & $\begin{array}{l}\text { Latency } \\
\text { period }\end{array}$ & $\begin{array}{l}\text { Duration of } \\
\text { denosumab }\end{array}$ & $\begin{array}{l}\text { Malignancy } \\
\text { present } \\
\text { prior to } \\
\text { denosumab }\end{array}$ & $\begin{array}{l}\text { Malignancy } \\
\text { outcome }\end{array}$ \\
\hline y) $10(50-59$ & $\begin{array}{l}\text { Recurrent } \\
\text { unresectable }\end{array}$ & Sacrum & $\begin{array}{l}\text { Diagnosis: High grade } \\
\text { undifferentiated spindle cell } \\
\text { sarcoma, consistent with } \\
\text { differentiation arising in } \\
\text { malignant GCTB }\end{array}$ & $\begin{array}{l}\text { Yes, } 25 \\
\text { fractions }\end{array}$ & $\begin{array}{l}6 y, 7 \\
\mathrm{mo}\end{array}$ & $1 \mathrm{y}, 8 \mathrm{mo}$ & Yes & $\begin{array}{l}\text { Surgery (resection } \\
\text { and curettage, } \\
\text { laminectomy); } \\
\text { deceased } 2 \text { mo after } \\
\text { malignancy } \\
\text { diagnosis }\end{array}$ \\
\hline \multicolumn{9}{|c|}{ Sarcomatous transformation } \\
\hline y) $11(30-39$ & $\begin{array}{l}\text { Recurrent } \\
\text { unresectable }\end{array}$ & $\begin{array}{l}\text { Distal } \\
\text { femur }\end{array}$ & $\begin{array}{l}\text { Diagnosis: Undifferentiated } \\
\text { spindle cell sarcoma }\end{array}$ & No & $11 y$ & $6 \mathrm{y}, 2 \mathrm{mo}$ & No & $\begin{array}{l}\text { Amputation and } \\
\text { chemotherapy; alive } \\
\text { at last follow-up }\end{array}$ \\
\hline y) $12(60-69$ & $\begin{array}{l}\text { Primary } \\
\text { resectable }\end{array}$ & Tibia & $\begin{array}{l}\text { Diagnosis: High-grade } \\
\text { osteosarcoma }\end{array}$ & No & $\begin{array}{l}2 y, 11 \\
\text { mo }\end{array}$ & $2 \mathrm{y}, 2 \mathrm{mo}$ & No & $\begin{array}{l}\text { Chemotherapy and } \\
\text { amputation; } \\
\text { deceased } 7 \text { mo after } \\
\text { malignancy } \\
\text { diagnosis }\end{array}$ \\
\hline y) $13(30-39$ & $\begin{array}{l}\text { Recurrent } \\
\text { resectable }\end{array}$ & $\begin{array}{l}\text { Distal } \\
\text { femur }\end{array}$ & $\begin{array}{l}\text { Diagnosis: Undifferentiated } \\
\text { spindle cell sarcoma }\end{array}$ & No & $\begin{array}{l}4 y, 9 \\
\text { mo }\end{array}$ & $2 \mathrm{y}, 5.5 \mathrm{mo}$ & No & $\begin{array}{l}\text { Tumor resection and } \\
\text { prosthesis plus } \\
\text { chemotherapy; alive, } \\
\text { no evidence of } \\
\text { disease at last } \\
\text { follow-up }\end{array}$ \\
\hline y) $14(50-59$ & $\begin{array}{l}\text { Primary } \\
\text { resectable }\end{array}$ & $\begin{array}{l}\text { Distal } \\
\text { femur }\end{array}$ & $\begin{array}{l}\text { Microscopic picture } \\
\text { corresponds to } \\
\text { chondroblastic/osteoblastic } \\
\text { osteosarcoma high grade that } \\
\text { probably developed from } \\
\text { GCTB } \\
\text { Diagnosis: High-grade } \\
\text { osteosarcoma }\end{array}$ & No & $\begin{array}{l}1 \mathrm{y}, 5 \\
\mathrm{mo}\end{array}$ & $\begin{array}{l}1 \mathrm{y}, 2 \text { mo; } \\
\text { progression } \\
\text { after only } 3 \\
\text { mo of } \\
\text { treatment }\end{array}$ & No & $\begin{array}{l}\text { Amputation plus } \\
\text { chemotherapy; alive } \\
\text { at last follow-up }\end{array}$ \\
\hline
\end{tabular}

$A B C$ aneurysmal bone cyst, GCTB giant cell tumor of bone, Gy Gray unit, mo months, SMGCTB secondary malignant giant cell tumor of bone, y years

${ }^{a}$ Patient numbers and age ranges (in brackets), instead of age at treatment, are identifiers for the purposes of this publication only and do not link to patients ${ }^{b}$ Malignancy likely, but not definitively, present prior to denosumab due to lack of sufficient biopsies for expert review; opinion of expert reviewers based on available evidence (existing biopsy samples or local pathologist report) as noted in the "malignant histology" column)

\section{Sarcomatous transformation of GCTB}

Four patients had malignancy deemed as true sarcomatous transformation (ie, not previously treated for GCTB) (Table 3). Patients with sarcomatous transformation presented with GCTB in the femur or tibia. Two patients had pathologic fracture at presentation (Patients 11 and 12). Unlike many cases of primary or SMGCTB, sarcomatous transformation was not present before receiving denosumab for all four patients. The time from diagnosis of GCTB to diagnosis of sarcomatous transformation ranged from 17 months to 11 years. Patients received denosumab from 14 months to 6 years, with the time from initial GCTB diagnosis to initiation of denosumab ranging from 2 months to 4 years.

\section{Malignancies misdiagnosed as benign GCTB}

A summary of the six patients determined to have misdiagnosis of benign GCTB is presented in Table 4. Two of these patients had lung nodules as the primary target lesion at the time of study enrollment (Patients 17 and 19); most patients were in the recurrent unresectable cohort. After re-examination, the correct diagnoses were undifferentiated spindle cell sarcoma (2 patients) and giant cell-rich osteosarcoma, pleomorphic rhabdomyosarcoma, osteogenic sarcoma, and phosphaturic mesenchymal tumor of mixed connective tissue type (1 patient each). Of note, two of these rare diagnoses included second pathologic opinions by global experts outside of the panel. Four patients were pathologically confirmed to have the malignancy present before denosumab treatment (Patients 17-20); for the other two (Patients 15 and 16), the malignancy was classified as likely, but not definitively, present before denosumab, based on imaging and clinical history, but it was not possible to confirm pathologically given that there were no available specimens or lack of adequate specimens for pathologic re-evaluation.

\section{Discussion}

Patients rarely develop malignancy in GCTB. In this study, the overall incidence of an adverse event of 

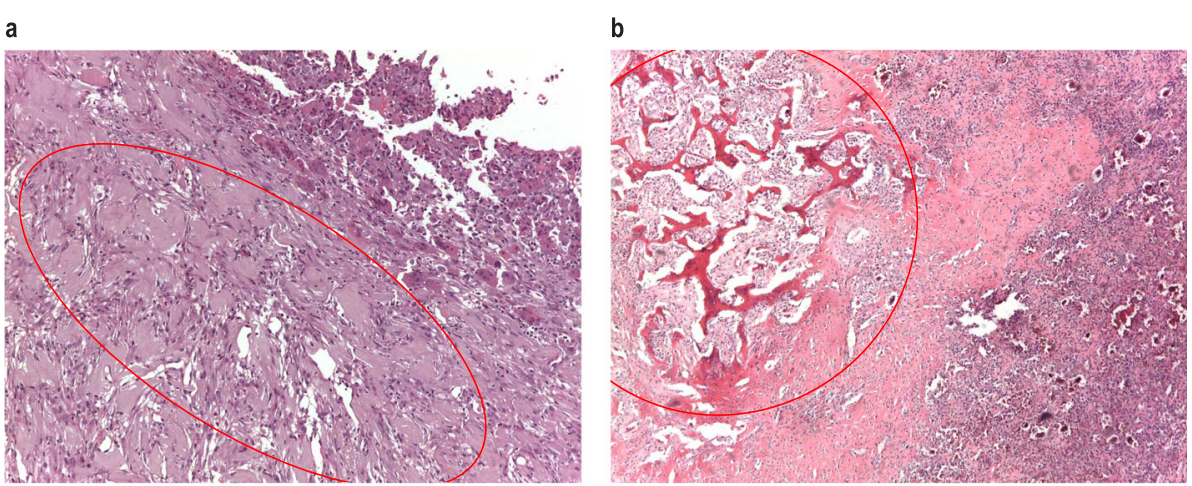

Fig. 4 Histologic features of malignancy in GCTB. a Primary malignant GCTB, pre-denosumab: proliferation of ovoid to spindle bland-appearing cells, with scattered reactive multinucleated osteoclast-like giant cells (top right of image), consistent with GCTB, juxtaposed to a proliferation of atypical spindle and pleomorphic cells, growing in fascicles, consistent with undifferentiated pleomorphic sarcoma (red circle). b Secondary malignant GCTB, pre-denosumab, (recurrence in 2008): histological features consistent with GCTB (bottom right of image), juxtaposed to a proliferation of atypical spindle cells, infiltrating in between the host bony trabeculae, consistent with high-grade undifferentiated spindle cell sarcoma (red circle)

new malignancy in GCTB was 3.8\% (20/526): five (1.0\%) had PMGCTB, five (1.0\%) had SMGCTB, four $(0.8 \%)$ had sarcomatous transformation, and six $(1.0 \%)$ had a misdiagnosis of benign GCTB. This malignancy rate in GCTB during the study, including sarcomatous transformation, was not higher than historical rates (range 1-15\%) [7, 8, 12-17]. A recent review of 2315 patients with GCTB in the pre-denosumab era indicated that the cumulative rate of malignancy was $4.0 \%$; the cumulative incidences of primary and SMGCTB were 1.6 and 2.4\%, respectively; these rates are comparable to the present study [5].

Historically, malignancies in GCTB are secondary malignancies typically observed after radiotherapy $[8,18]$, but they may also occur after surgical treatment, such as bone grafts, without adjuvant radiotherapy [9]. Notably, one patient categorized as having SMGCTB did not receive radiotherapy: this categorization was based on the fact that malignancy was present prior to denosumab therapy. Primary malignancies are considered to be rare [7]. There have been some observations of sarcoma development in patients with GCTB treated with denosumab in a phase 2 trial, none of which were thought to be caused by denosumab [11]. However, as concern has been expressed about the possible risk of malignant transformation associated with denosumab, the best approach is to assess this risk in the context of potential complications introduced by misdiagnosis [19]. Diagnosis of malignancy in GCTB can be challenging because radiologic features of primary malignancy are often identical to those of benign GCTB [6, 7]. Furthermore, there is a high level of

Table 4 Misdiagnosis of benign GCTB

\begin{tabular}{|c|c|c|c|c|c|}
\hline $\begin{array}{l}\text { Patient no. } \\
\text { (age range) }\end{array}$ & Cohort & $\begin{array}{l}\text { Site at } \\
\text { enrollment }\end{array}$ & Malignant histology & $\begin{array}{l}\text { Malignancy present } \\
\text { prior to denosumab? }\end{array}$ & Malignancy outcome \\
\hline $15(20-29 y)$ & $\begin{array}{l}\text { Primary } \\
\text { resectable }\end{array}$ & Distal femur & Giant cell-rich osteosarcoma & Likely $^{\mathrm{b}}$ & $\begin{array}{l}\text { Amputation; deceased } 12 \text { mo } \\
\text { after malignancy diagnosis }\end{array}$ \\
\hline $16(70-79 y)$ & $\begin{array}{l}\text { Recurrent } \\
\text { unresectable }\end{array}$ & Pubic ramus & Pleomorphic rhabdomyosarcoma & Likely $^{\mathrm{b}}$ & $\begin{array}{l}\text { Deceased } 6 \text { mo after malignancy } \\
\text { diagnosis }\end{array}$ \\
\hline $17(40-49 y)$ & $\begin{array}{l}\text { Recurrent } \\
\text { unresectable }\end{array}$ & Lung & $\begin{array}{l}\text { Undifferentiated spindle cell } \\
\text { sarcoma }\end{array}$ & Yes & $\begin{array}{l}\text { Deceased } 4 \text { mo after malignancy } \\
\text { diagnosis }\end{array}$ \\
\hline $18(20-29 y)$ & $\begin{array}{l}\text { Recurrent } \\
\text { unresectable }\end{array}$ & Femur & $\begin{array}{l}\text { Osteogenic sarcoma (present pre- } \\
\text { enrollment) }\end{array}$ & Yes & $\begin{array}{l}\text { Chemotherapy and resection of } \\
\text { tumor; alive at last follow-up }\end{array}$ \\
\hline $19(10-19 y)$ & $\begin{array}{l}\text { Recurrent } \\
\text { unresectable }\end{array}$ & $\begin{array}{l}\text { Lung and } \\
\text { cuneiform bones } \\
\text { of foot }\end{array}$ & $\begin{array}{l}\text { Phosphaturic mesenchymal tumor } \\
\text { of mixed connective tissue type }\end{array}$ & Yes & $\begin{array}{l}\text { Alive at last follow-up with } \\
\text { progressive disease }\end{array}$ \\
\hline $20(30-39 y)$ & $\begin{array}{l}\text { Recurrent } \\
\text { resectable }\end{array}$ & Humerus & $\begin{array}{l}\text { Undifferentiated spindle cell } \\
\text { sarcoma }\end{array}$ & Yes & Alive at last follow-up \\
\hline
\end{tabular}

GCTB giant cell tumor of bone, mo months, $y$ years

${ }^{a}$ Patient numbers and age ranges (in brackets), instead of age at treatment, are identifiers for the purposes of this publication only and do not link to patients ${ }^{b}$ Malignancy likely, but not definitively, present prior to denosumab due to lack of sufficient biopsies for expert review; opinion of expert reviewers based on available evidence 
heterogeneity among primary malignant tumors [6]. As a result, primary malignancies may only be observed after re-examination. We also found six misdiagnoses; this is not a surprising finding as these malignancies, along with GCTB, contain giant cells and are rare and therefore difficult to diagnose. The lack of clear diagnostic criteria for malignant GCTB further complicates diagnosis. From a pathological point of view, the main feature that represents a potential misdiagnosis is the presence of a giant cell component that can be present in other malignant mesenchymal tumors. Other signs of potential misdiagnosis of GCTB may include poor mineralization or rapid relapse in pain or no pain relief during treatment with denosumab. The use of comprehensive histologic sampling, careful follow-up, and timely treatment of local recurrence is therefore recommended [5].

In our study, $67 \%$ of the nine malignancies with evaluable imaging showed poor mineralization with denosumab treatment and in many cases were also accompanied by a rapid relapse in pain or a failure of the typical dramatic improvement in pain response that is usually observed with denosumab initiation [11]. Mineralization is an early indication of response and an expected finding of denosumab treatment based on its mechanism of action; therefore, in cases where denosumab treatment does not lead to adequate pain control or demonstrates less than anticipated tumor mineralization, clinical reassessment is suggested with consideration for potential misdiagnosis and re-biopsy of the lesion [20].

Currently, there are no well-established tumor response criteria for patients with GCTB [21]. Radiological classification based on computed tomography (CT) images may be more accurate than Choi criteria in identifying early tumor changes due to denosumab therapy [22]. Based on post hoc analysis of patients with GCTB treated with denosumab, increased positron-emission tomography avidity may accurately identify malignant changes [20]. Clinical presentation is important to distinguish patients without symptoms or pain, as well as those with increasing symptoms. Routine follow-up with CT with comparison to all prior imaging, including baseline studies, is essential. The interval between imaging should be left to physician's discretion according to patient characteristics.

Difficulties exist in distinguishing normal postdenosumab bone histopathology changes from malignant changes. Histopathologic changes associated with denosumab in GCTB include a total or near-total disappearance of osteoclast-like giant cells; residual tumor cells are primarily normal-appearing spindle cells arranged in fascicles often with storiform pattern [23]. Osteoid production with variable degree of mineralization is typically present [23]. A case report of a patient with GCTB treated with nine cycles of denosumab showed pseudosarcomatous spindle cell proliferation with osteoid matrix resembling osteosarcoma [24]. Such observations after denosumab treatment further complicate proper diagnosis of secondary malignancies.

Only seven patients had both pre- and postdenosumab immunohistochemistry available. It is interesting to observe an increase in nuclear expression of P53 in secondary GCTB or sarcomatous transformation, which has been previously implicated in malignant transformation of GCTB [6, 25]. H3F3A encodes the replication-independent histone $\mathrm{H} 3.3 ; 49$ to $92 \%$ of patients with GCTB have mutations in $H 3 F 3 A$ (typically G34W); chondroblastoma has been associated with mutations in $H 3 F 3 B$; other giant cell containing tumors had no or few mutations in either of these genes [26-28]. One patient with PMGCTB (Patient 2) and one with a misdiagnosis (Patient 16) who had undifferentiated pleomorphic sarcoma were negative for $H 3 F 3 A$ mutations. Because of the high rate of $H 3 F 3 A$ mutations in GCTB, patients negative for mutations should be suspected of having other bone tumor types and followed closely. One case (Patient 13) showed MDM2 amplification in the malignant transformation but not in the initial GCTB diagnosis, suggesting a possible role of MDM2 amplification in the development of malignancy.

Fifteen of the 20 malignancies were definitively or likely present before denosumab was initiated, which excludes any potential involvement of denosumab in these cases. Denosumab has established safety through several clinical studies in patients with GCTB and in patients with solid tumors used for prevention of skeletal-related events [29-31]. The RANK/RANKL pathway is also known to be involved in tumorigenesis [32, 33]. GCTB consists of stromal cells expressing RANKL and osteoclast-like giant cells expressing the RANK receptor, and signaling through the RANK receptor contributes to osteolysis and tumor growth [31, 34]. Denosumab blocks RANKL from binding to its receptor on the surface of osteoclasts, their precursors, and osteoclast-like giant cells [20]. Based on this, it is highly unlikely that denosumab would increase the risk of malignancy [35]. The incidence of malignancy was not higher than historical rates, which also supports no involvement of denosumab.

\section{Conclusions}

In conclusion, we identified 20 malignancies in an openlabel study, for an overall rate of $3.8 \%$, which is not higher than historical rates. Out of those malignancies, six were misdiagnoses of benign GCTB. Furthermore, 15 out of 20 malignancies were definitively or likely present before denosumab was initiated, excluding any potential involvement of denosumab in these cases. Given the 
rarity of this complication in the already rare GCTB, careful diagnosis and follow-up are recommended.

\section{Supplementary Information}

The online version contains supplementary material available at https://doi. org/10.1186/s12885-020-07739-8.

\section{Additional file 1}

\section{Abbreviations}

CT: Computed tomography; GCTB: Giant cell tumor of bone; MDM2: Mouse double minute 2; PM: Primary malignant; PMGCTB: Primary malignant giant cell tumor of bone; SM: Secondary malignant; SMGCTB: Secondary malignant giant cell tumor of bone

\section{Acknowledgements}

The authors wish to acknowledge Miranda Tradewell and Rick Davis (ICON plc, North Wales, PA), whose work was funded by Amgen Inc., and Albert Rhee (Amgen Inc., Thousand Oaks, CA) for assistance with the writing of this manuscript.

\section{Authors' contributions}

EP participated in study concept and/or design, and contributed to acquisition, analysis, or interpretation of data. LS participated in study concept and/or design, contributed to acquisition, analysis, or interpretation of data, and provided administrative, technical, or material support. MG contributed to acquisition, analysis, or interpretation of data, and provided administrative, technical, or material support. AR contributed to acquisition, analysis, or interpretation of data, and provided administrative, technical, or material support. PR participated in study concept and/or design, and contributed to acquisition, analysis, or interpretation of data. SB participated in study concept and/or design, and contributed to acquisition, analysis, or interpretation of data. J-YB participated in study concept and/or design, and contributed to acquisition, analysis, or interpretation of data. TD contributed to acquisition, analysis, or interpretation of data, and provided statistical analysis of the data. DJ contributed to acquisition, analysis, or interpretation of data, and provided statistical analysis of the data. PP participated in study concept and/or design, contributed to acquisition, analysis, or interpretation of data, and provided administrative, technical, or material support (imaging/ pathology review).

All authors were involved in drafting the manuscript and critical revision of the manuscript for important intellectual content. All authors read and approved the final manuscript and agree to be accountable for all aspects of the work and to ensure that questions related to the accuracy or integrity of the manuscript are appropriately investigated and resolved.

\section{Funding}

This study was funded by Amgen Inc. Amgen Inc. participated in and supported the design and conduct of the study and the collection and interpretation of the data. The decision to submit the manuscript for publication was made by the authors.

\section{Availability of data and materials}

Qualified researchers may request the dataset supporting the conclusions of this article from Amgen. Complete details are available at the following: https://www.amgen.com/science/clinical-trials/clinical-data-transparencypractices/clinical-trial-data-sharing-request/

\section{Ethics approval and consent to participate}

The study is registered at clinicaltrials.gov (number NCT00680992) and was performed at 30 sites in 12 countries. The study was approved by each site's independent ethics committee, and all patients provided written informed consent.

\section{Consent for publication}

Not applicable

\section{Competing interests}

Dr. Palmerini has served on advisory boards for Amgen, Daiichi Sankyo, Lilly, EUSA Pharma, and Deciphera; has received other research support from Bristol-Myers Squibb, Pfizer, Daiichi Sankyo, and PharmaMar; and has received travel support from Lilly, PharmaMar, and Takeda.

Dr. Seeger reports nothing to disclose.

Drs. Gambarotti, Righi, and Picci received institutional funding from Amgen for clinical and pathological input and revision of the cases reported in this study.

Dr. Reichardt has received personal fees from Novartis, Pfizer, Bayer, PharmaMar, Clinigen, Lilly, Deciphera, Merck, Roche, and Amgen Inc. Dr. Bukata has served as a consultant for Amgen and Radius, speakers's bureau for Radius, and board service for the Orthopaedic Research Society and the US National Osteoporosis Foundation.

Dr. Blay has received research grants and honoraria from Novartis and Amgen Inc.

Drs. Dai and Jandial are employed by and own stock in Amgen Inc.

\section{Author details}

${ }^{1}$ Chemotherapy Unit, IRCCS Istituto Ortopedico Rizzoli, Department of Experimental, Diagnostic and Specialty Medicine (DIMES), Bologna University, 40136 Bologna, Italy. ${ }^{2}$ David Geffen School of Medicine, UCLA Health System, 200 UCLA Medical Plaza Suite 165-57, Los Angeles, CA 90095, USA. ${ }^{3}$ Department of Pathology, IRCCS Istituto Ortopedico Rizzoli, Bologna, Italy. ${ }^{4}$ Department of Interdisciplinary Oncology, Sarcoma Center Berlin-Brandenburg; HELIOS Klinikum Berlin-Buch, Schwanebecker Chaussee 50, 13125 Berlin, Germany. ${ }^{5}$ Department of Medical Oncology, Leon Berard Center, 28, rue Laennec 269373 Lyon Cedex 08, Lyon, France. ${ }^{6} \mathrm{Global}$ Development (Oncology), Amgen Inc., One Amgen Center Drive, MS 38-2-B, Thousand Oaks, CA 91320-1799, USA.

Received: 1 October 2020 Accepted: 15 December 2020

Published online: 22 January 2021

\section{References}

1. Mendenhall WM, Zlotecki RA, Scarborough MT, Gibbs CP, Mendenhall NP. Giant cell tumor of bone. Am J Clin Oncol. 2006;29(1):96-9.

2. Alberghini M, Kliskey K, Krenacs T, Picci P, Kindblom L, Forsyth R, et al. Morphological and immunophenotypic features of primary and metastatic giant cell tumour of bone. Virchows Arch. 2010;456(1):97-103.

3. NCCN Clinical Practice Guidelines in Oncology (NCCN Guidelines ${ }^{\circledast}$ ) Bone Cancer. Version 1. 2018. https://www.nccn.org/professionals/physician_gls/ PDF/bone.pdf.

4. Flanagan A, Larousserie F, O'Donnel P. Giant cell tumor of bone. In: WHO classification of tumours, Edited by WHO Classification of Tumours Editorial Board. 5th ed. Lyon: International Agency for Research on Cancer; 2020. p. 440-6.

5. Palmerini E, Picci P, Reichardt P, Downey G. Malignancy in giant cell tumor of bone: a review of the literature. Technol Cancer Res Treat. 2019;18: 1533033819840000

6. Gong L, Liu W, Sun X, Sajdik C, Tian X, Niu X, et al. Histological and clinical characteristics of malignant giant cell tumor of bone. Virchows Arch. 2012; 460(3):327-34

7. Bertoni F, Bacchini P, Staals EL. Malignancy in giant cell tumor of bone. Cancer. 2003;97(10):2520-9.

8. Rock MG, Sim FH, Unni KK, Witrak GA, Frassica FJ, Schray MF, et al. Secondary malignant giant-cell tumor of bone. Clinicopathological assessment of nineteen patients. J Bone Joint Surg Am. 1986;68(7):1073-9.

9. Unni KK. How to diagnose malignant giant cell tumor. AJSP: Rev Rep. 2001; 6(1):33-7.

10. XGEVA ${ }^{\oplus}$. In: Denosumab prescribing information. Thousand Oaks: Amgen Inc.; 2020.

11. Chawla S, Blay JY, Rutkowski P, Le Cesne A, Reichardt P, Gelderblom H, et al. Denosumab in patients with giant-cell tumour of bone: a multicentre, open-label, phase 2 study. Lancet Oncol. 2019;20(12):1719-29.

12. Mondal A, Kundu B, Gupta S, Biswas J. Secondary malignant giant cell tumour of bone--a study of five cases with short review of literature. Indian J Pathol Microbiol. 2002;45(3):273-5.

13. Boriani S, Sudanese A, Baldini N, Picci P. Sarcomatous degeneration of giant cell tumours. Ital J Orthop Traumatol. 1986;12(2):191-9. 
14. Campanacci M, Baldini N, Boriani S, Sudanese A. Giant-cell tumor of bone. J Bone Joint Surg Am. 1987;69(1):106-14.

15. Domovitov SV, Healey JH. Primary malignant giant-cell tumor of bone has high survival rate. Ann Surg Oncol. 2010;17(3):694-701.

16. Anract $P$, De Pinieux $G$, Cottias $P$, Pouillart $P$, Forest $M$, Tomeno B. Malignant giant-cell tumours of bone. Clinico-pathological types and prognosis: a review of 29 cases. Int Orthop. 1998;22(1):19-26.

17. Unni KK, Inwards CY. Dahlin's bone tumours: general aspects and data on 10165 cases. 6th ed. Philadelphia: Lippincott Williams \& Wilkins; 2010.

18. Skubitz KM. Giant cell tumor of bone: current treatment options. Curr Treat Options in Oncol. 2014;15(3):507-18.

19. Healey JH. Denosumab for giant cell tumour of bone: success and limitations. Lancet Oncol. 2019;20(12):1627-8.

20. Engellau J, Seeger L, Grimer R, Henshaw R, Gelderblom H, Choy E, et al. Assessment of denosumab treatment effects and imaging response in patients with giant cell tumor of bone. World J Surg Oncol. 2018;16:191.

21. Ueda T, Morioka H, Nishida Y, Kakunaga S, Tsuchiya H, Matsumoto Y, et al. Objective tumor response to denosumab in patients with giant cell tumor of bone: a multicenter phase II trial. Ann Oncol. 2015;26(10):2149-54.

22. Campanacci L, Sambri A, Medellin MR, Cimatti P, Errani C, Donati DM. A new computerized tomography classification to evaluate response to denosumab in giant cell tumors in the extremities. Acta Orthop Traumatol Turc. 2019;53(5):376-80.

23. Girolami I, Mancini I, Simoni A, Baldi GG, Simi L, Campanacci D, et al. Denosumab treated giant cell tumour of bone: a morphological, immunohistochemical and molecular analysis of a series. J Clin Pathol. 2016; 69(3):240-7.

24. Santosh N, Mayerson JL, Iwenofu OH. Pseudosarcomatous spindle cell proliferation with osteoid matrix mimicking osteosarcoma: a distinct histologic phenotype in giant cell tumor of bone following denosumab therapy. Appl Immunohistochem Mol Morphol. 2016;24(3):e18-9.

25. Okubo T, Saito T, Mitomi H, Takagi T, Torigoe T, Suehara Y, et al. p53 mutations may be involved in malignant transformation of giant cell tumor of bone through interaction with GPX1. Virchows Arch. 2013;463(1):67-77.

26. Behjati S, Tarpey PS, Presneau N, Scheipl S, Pillay N, Van Loo P, et al. Distinct $\mathrm{H} 3 \mathrm{~F} 3 \mathrm{~A}$ and $\mathrm{H} 3 \mathrm{~F} 3 \mathrm{~B}$ driver mutations define chondroblastoma and giant cell tumor of bone. Nat Genet. 2013;45(12):1479-82.

27. Cleven AH, Hocker S, Briaire-de Bruijn I, Szuhai K, Cleton-Jansen AM, Bovee JV. Mutation analysis of H3F3A and $\mathrm{H} 3 \mathrm{~F} 3 \mathrm{~B}$ as a diagnostic tool for giant cell tumor of bone and chondroblastoma. Am J Surg Pathol. 2015;39(11):157683.

28. Nohr E, Lee LH, Cates JM, Perizzolo M, Itani D. Diagnostic value of histone 3 mutations in osteoclast-rich bone tumors. Hum Pathol. 2017;68:119-27.

29. Chawla S, Henshaw R, Seeger L, Choy E, Blay JY, Ferrari S, et al. Safety and efficacy of denosumab for adults and skeletally mature adolescents with giant cell tumour of bone: interim analysis of an open-label, parallel-group, phase 2 study. Lancet Oncol. 2013;14(9):901-8.

30. Thomas D, Henshaw R, Skubitz K, Chawla S, Staddon A, Blay JY, et al. Denosumab in patients with giant-cell tumour of bone: an open-label, phase 2 study. Lancet Oncol. 2010;11(3):275-80.

31. Branstetter DG, Nelson SD, Manivel JC, Blay JY, Chawla S, Thomas DM, et al. Denosumab induces tumor reduction and bone formation in patients with giant-cell tumor of bone. Clin Cancer Res. 2012;18(16):4415-24.

32. Sisay M, Mengistu G, Edessa D. The RANK/RANKL/OPG system in tumorigenesis and metastasis of cancer stem cell: potential targets for anticancer therapy. Onco Targets Ther. 2017;10:3801-10.

33. Raje NS, Bhatta S, Terpos E. Role of the RANK/RANKL pathway in multiple myeloma. Clin Cancer Res. 2019;25(1):12-20.

34. Roodman GD. Mechanisms of bone metastasis. N Engl J Med. 2004;350(16): $1655-64$.

35. Coleman R, Finkelstein DM, Barrios C, Martin M, Iwata H, Hegg R, et al. Adjuvant denosumab in early breast cancer (D-CARE): an international, multicentre, randomised, controlled, phase 3 trial. Lancet Oncol. 2020;21(1): $60-72$.

\section{Publisher's Note}

Springer Nature remains neutral with regard to jurisdictional claims in published maps and institutional affiliations.

\section{Ready to submit your research? Choose BMC and benefit from:}

- fast, convenient online submission

- thorough peer review by experienced researchers in your field

- rapid publication on acceptance

- support for research data, including large and complex data types

- gold Open Access which fosters wider collaboration and increased citations

- maximum visibility for your research: over $100 \mathrm{M}$ website views per year

At BMC, research is always in progress.

Learn more biomedcentral.com/submissions 